\title{
Improved safety and efficacy of a lipid emulsion loaded with a paclitaxel-cholesterol complex for the treatment of breast tumors
}

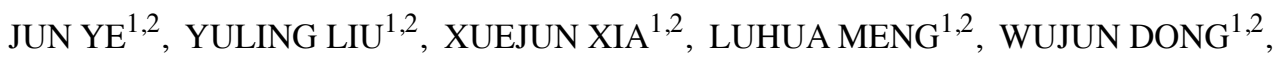 \\ RENYUN WANG ${ }^{1,2}$, ZHAODI FU ${ }^{1,3}$, HONGYAN LIU ${ }^{1,3}$ and RUI HAN ${ }^{1,3}$
}

\author{
${ }^{1}$ State Key Laboratory of Bioactive Substance and Function of Natural Medicines; ${ }^{2}$ Beijing Key Laboratory of \\ Drug Delivery Technology and Novel Formulation; ${ }^{3}$ Department of Pharmacology, Institute of Materia Medica, \\ Chinese Academy of Medical Sciences \& Peking Union Medical College, Beijing 100050, P.R. China
}

Received January 18, 2016; Accepted February 14, 2016

DOI: $10.3892 /$ or.2016.4787

\begin{abstract}
The aim of the present study was to develop a lipid emulsion loaded with a paclitaxel-cholesterol complex (PTX-CH Emul) in order to improve the safety and efficacy of paclitaxel (PTX) and evaluate its antitumor activity against commercially available formulation $\operatorname{Taxol}^{\circledR}$. PTX-CH Emul resembling a low density lipoprotein lipid structure, exhibited an ideal particle size, high drug loading capability, high drug encapsulation efficiency and excellent stability. PTX-CH Emul showed superior in vitro anticancer efficacy against triple-negative MDA-MB-231 breast cancer cells when compared with a paclitaxel emulsion (PTX Emul) and Taxol. The $\mathrm{IC}_{70}$ value of PTX-CH Emul was almost 1.5- and 2.4-fold lower than that of PTX Emul and Taxol, respectively. Compared with PTX Emul and Taxol, PTX-CH Emul exhibited stronger and more rapid inhibitory effects on 3D tumor spheroids of MDA-MB-231 cells. Additionally, in vivo tumor-targeting study showed that PTX-CH Emul had higher specificity and efficiency in intratumoral accumulation as compared to PTX Emul. Finally, the maximum tolerated dose (MTD) of PTX-CH Emul was 2.25-fold higher than that of Taxol, suggesting that PTX-CH Emul exhibited better safety profiles in vivo than Taxol. At the MTDs, PTX-CH Emul exhibited superior antitumor efficacy in nude mice bearing MDA-MB-231 xenografts in comparison to Taxol. Therefore, PTX-CH Emul as reported here showed high potential as a drug carrier for PTX in clinical applications involving the targeting of triple-negative breast cancer.
\end{abstract}

Correspondence to: Professor Yuling Liu or Dr Xuejun Xia, State Key Laboratory of Bioactive Substance and Function of Natural Medicines, Institute of Materia Medica, Chinese Academy of Medical Sciences \& Peking Union Medical College, 1 Xiannongtan Street, Beijing 100050, P.R. China

E-mail: ylliu@imm.ac.cn

E-mail:xjxia@imm.ac.cn

Key words: lipid emulsion, paclitaxel-cholesterol complex, tumor targeting, breast tumor

\section{Introduction}

Paclitaxel (PTX) is the first of a class of microtubule-stabilizing agents and has significant antitumor activity in clinical treatments against a broad range of solid tumors, especially against breast, lung and ovarian cancer $(1,2)$. The first injectable dosage form of PTX is Taxol $^{\circledR}$, which is composed of a mixture of Cremophor ${ }^{\circledR}$ EL and dehydrated alcohol (1:1, $\mathrm{v} / \mathrm{v}$ ) due to its low solubility. This vehicle may result in a variety of side effects such as hypersensitivity, nephrotoxicity and neurotoxicity, attributed mainly to Cremophor EL $(3,4)$. To overcome the problems caused by Cremophor EL and improve the drug efficacy, a variety of formulations have been investigated to administer PTX in a more safe and convenient manner, including liposomes, emulsions, nanoparticles and polymeric micelles (5-8). However, two common drawbacks of most nanomedicines which limit their clinical use are that the materials used are potentially toxic/allergic and that they are unable to be up-scaled by industrialized production $(9,10)$.

Lipid emulsion offers potential advantages for the delivery of poorly water-soluble drugs owing to many favorable properties, such as nanometer size range with larger surface area, manufacturing with biocompatible materials, and easy production on a large scale $(11,12)$. Furthermore, lipid emulsion is a commonly acceptable formulation used in the clinic for years. Over the last decade, several studies reported on the benefits of a cholesterol-rich emulsion termed LDE to deliver therapeutic agents to cancers (13-16). The LDE resembles the low density lipoprotein (LDL) lipid portion structure and binds to LDL receptors on the cancer cell surface. Increased LDL requirements and LDL receptor numbers have been observed in many types of tumors, including breast, human glioblastoma and lung tumors (17-19). Although the LDE has been confirmed to possess tumor-targeting effects mediated by LDL receptors and shows good drug loading capacity of PTX, it is stable only 8 days at $4^{\circ} \mathrm{C}$ (13). The instability of LDE-PTX, which may be attributed to the poor lipophilicity of PTX (14), makes it less promising for clinical evaluation.

Previous efforts on improving its stability have been focused on developing the paclitaxel-cholesterol prodrug with increased lipophilicity $(20,21)$. What we found is that the lipid emulsion loaded with the paclitaxel-cholesterol prodrug 
exhibited long-term stability and high intratumoral accumulation (20), but the in vivo antitumor effect was greatly reduced in comparision to Taxol (unpublished data), which may attribute to the low concentration of PTX released from the paclitaxel-cholesterol prodrug by in vivo enzymatic cleavage. Alternatively, a paclitaxel-cholesterol complex connected by intermolecular hydrogen bonds was prepared and a novel lipid emulsion (PTX-CH Emul), which was composed of a paclitaxel-cholesterol complex surrounded by a phospholipid monolayer, was engineered in our previous study (22). Compared with the tumor-targeting LDE-PTX loaded with PTX and cholesteryl ester reported previously (13), substitution of the paclitaxel-cholesterol complex for PTX and cholesteryl ester of LDE-PTX would be doubly advantageous: the paclitaxel-cholesterol complex not only greatly improves PTX solubility in the oil phase, but can also function as the component of LDL-cholesterol. The resulting lipid emulsion survives autoclaving and is more stable than LDE-PTX (12 months at $6^{\circ} \mathrm{C}$ vs. 8 days at $4^{\circ} \mathrm{C}$ ). The long-term stability provides conditions for further clinical treatment. Additionally, combined with the particularly abundant expression profile of the LDL receptors in breast tumor cells (19), we speculate that the PTX-CH Emul that mimics the receptor-mediated binding and uptake properties of LDL could act as an ideal breast tumor-targeting PTX delivery system.

In this study, we prepared a PTX-CH Emul and investigated its antitumor activity by comparing it to a conventional PTX-loaded lipid emulsion (PTX Emul) and Taxol in vitro and in vivo using breast tumor models, including $2 \mathrm{D}$ monolayer tumor cells, 3D multicellular tumor spheroids and xenograft tumor models. Because PTX is used to treat patients with triplenegative breast cancer (TNBC) and LDL receptor mRNA is more abundant in TNBC cells than in non-TNBC cells $(19,23)$, MDA-MB-231, a human TNBC cell line, was chosen for treatment with the 3 PTX formulations. Apart from comparing the antitumor activity, the tumor-targeting effect of PTX-CH Emul was also investigated by monitoring the intratumoral accumulation in xenograft tumor models. Interestingly, the results showed that our present study may provide a successful paradigm for the treatment of breast tumors and for further clinical applications.

\section{Materials and methods}

Materials. Paclitaxel and Taxol ${ }^{\circledR}$ were purchased from Beijing Union Pharmaceutical Factory (Beijing, China). RPMI-1640 medium, fetal bovine serum (FBS), $0.25 \%$ (w/v) trypsin solution and antibiotic agents were purchased from Invitrogen Life Technologies (Carlsbad, CA, USA). Phosphate-buffered saline (PBS, pH, 7.4) was purchased from Hyclone (Logan, UT, USA). Cell Counting Kit-8 (CCK-8) was obtained from Dojindo Laboratories (Tokyo, Japan). Nile red, fluorescein and Hoechst 33258 were procured from Sigma-Aldrich (St. Louis, MO, USA). DiR was obtained from AAT Bioquest, Inc. (Sunnyvale, CA, USA). All solvents such as acetone and acetonitrile were of analytical or high performance liquid chromatography (HPLC) grade and were used according to the manufacturer's instructions.

Preparation of the PTX-CH Emul. PTX-CH Emul was prepared using a solvent evaporation method and high-pres- sure homogenization, respectively, as previously reported (22). The PTX Emul was prepared using the same method except that the process of dissolving PTX in oil was different. Briefly, PTX was dissolved in acetone. Then soybean oil-medium chain triglyceride $(1: 1, \mathrm{w} / \mathrm{w})$ and vitamin $\mathrm{E}$ acetate were added to this organic phase. Acetone was removed by evaporation and the oily paste was used as the oil phase of emulsion. Fluorescein-labeled emulsion (FL emulsion) was prepared using the same procedure, except that fluorescein or the fluorescein-cholesterol complex was dissolved in oil in advance.

For the in vivo tumor-targeting imaging studies, DiR-encapsulated PTX-CH Emul and DiR-encapsulated PTX Emul were prepared using the same method as that of PTX-CH Emul and PTX Emul, respectively.

Characterization of PTX-CH Emul. The emulsion particle size was measured by a PSS NICOMP Particle Sizing System (PSS, Port Richey, FL, USA) after appropriate dilution with double-distilled water. The paclitaxel content of the emulsion was measured using an HPLC system with a UV detector (Agilent Technologies Inc., Cotati, CA, USA). The emulsion encapsulation efficiency was determined by measuring free paclitaxel in the aqueous phase.

Confocal laser scanning microscopy (CLSM) (FV1000; Olympus Corp., Tokyo, Japan) was employed to visualize the microenvironment of the major components in the emulsion, lipid based depot and lipid nanoparticles $(6,24)$. The fluorescent emulsions were labeled with two fluorescent probes, Nile red and fluorescein-cholesterol complex. Nile red- and fluorescein-labeled emulsions were prepared at a load ratio of $0.005 \%(\mathrm{w} / \mathrm{v})$, diluted with appropriate quantities of distilled water, and a droplet of the emulsion dispersion was then sealed between two coverslips for CLSM measurement.

Cell line and cell culture. MDA-MB-231 cells were purchased from the Department of Cell Resources, Institute of Basic Medical Sciences, Chinese Academy of Medical Sciences \& Peking Union Medical College (Beijing, China). The cell line was authenticated by the suppliers and cells were passaged in the laboratory. Cells were maintained in a $37^{\circ} \mathrm{C} / 5 \% \mathrm{CO}_{2}$ humidified chamber in RPMI-1640 media supplemented with $10 \% \mathrm{FBS}, 100 \mathrm{U} / \mathrm{ml}$ penicillin and $100 \mu \mathrm{g} / \mathrm{ml}$ streptomycin.

Animals and human tumor xenografts. Female BALB/c nude mice with a body weight of 21 to $25 \mathrm{~g}$ and ages ranging from 6 to 8 weeks were obtained from Beijing HFK Bioscience Co., Ltd. (Beijing, China). All animal protocols were approved by the Institutional Animal Care and Use Committee. Female $\mathrm{BALB} / \mathrm{c}$ nude mice were inoculated subcutaneously in the right armpits with $0.2 \mathrm{ml}\left(2 \times 10^{6}\right)$ MDA-MB-231 cell suspension. Cells were propagated in vivo as solid tumors. The tumors were implanted subcutaneously as fragments and allowed to grow to a median size of $100-200 \mathrm{~mm}^{3}$ before treatment was initiated.

In vitro cytotoxicity assay. The cytotoxicity of Blank Emul, PTX-CH Emul, PTX Emul and Taxol in the MDA-MB-231 cells was measured using CCK-8 (25). Cells were seeded in 96-well plates at a density of 3,000 cells/well. After $24 \mathrm{~h}$, the cells were incubated with PTX-CH Emul, PTX Emul or Taxol at different PTX concentrations. The sequence of Blank Emul 
concentrations was exactly the same as PTX-CH Emul. After $72 \mathrm{~h}$ of incubation, the number of viable cells was determined using CCK-8 according to the manufacturer's instructions. Untreated control cells were considered to be $100 \%$ viable. The concentration required for $50 \%$ or $70 \%$ growth inhibition $\left(\mathrm{IC}_{50}\right.$ or $\mathrm{IC}_{70}$ values, respectively) was determined with SPSS19.0 software (SPSS Inc., Chicago, IL, USA) as the mean \pm standard deviation (SD).

In vitro cellular uptake. MDA-MB-231 cells were seeded into 6 -well chamber slides and allowed to attach overnight. The medium was replaced with culture medium containing fluorescein-DMSO solution (FL-DMSO), FL emulsion or emulsion loaded with fluorescein-cholesterol complex (FL-CH emulsion). After incubation for $4 \mathrm{~h}$ at $37^{\circ} \mathrm{C}$, cells were washed three times with cold PBS, fixed with $4 \%$ paraformaldehyde (PFA), and the nuclei were counterstained using Hoechst 33258. The slides were observed using an Olympus FV1000 CLSM (26).

Growth inhibition of 3D breast tumor spheroids. Ex vitro 3D breast tumor spheroids of MDA-MB-231 cells were developed using a liquid overlay system (27). An agarose solution $(1.5 \%, \mathrm{w} / \mathrm{v})$ was prepared in serum-free RPMI-1640 media by heating at $80^{\circ} \mathrm{C}$ for $30 \mathrm{~min}$, followed by sterilization in an autoclave. Each well in the 96-well plates was coated with a thin layer $(50 \mu \mathrm{l})$ of the sterilized agarose solution. For optimal spheroid formation, 2,000 cells were added to each well and medium containing 2.5\% Matrigel (BD Biosciences, San Jose, CA, USA) was added. The plate was centrifuged for $10 \mathrm{~min}$ at $1000 \mathrm{x} \mathrm{g}$ and cultured in a standard cell incubator at $37^{\circ} \mathrm{C}$ with $5 \% \mathrm{CO}_{2}$. Cell culture medium was changed every 2 days. After 7 days, separate groups of spheroids were incubated with culture medium, PTX-CH Emul, PTX Emul or Taxol, each at the PTX concentration of $0.5 \mu \mathrm{g} / \mathrm{ml}$. Growth inhibition was monitored by measuring the size of the spheroids using an inverted phase microscope at $0,1,3,5$ and 7 days of treatment. The major $\left(\mathrm{d}_{\max }\right)$ and minor $\left(\mathrm{d}_{\min }\right)$ diameter of each spheroid was determined, and spheroid volume was calculated using the following formula: $\mathrm{V}=\left(\pi \times \mathrm{d}_{\max } \times \mathrm{d}_{\min }\right) / 6$. The spheroid volume ratio was calculated using the following formula: $\mathrm{R}$ $(\%)=\left(\mathrm{V}_{\text {dayi }} / \mathrm{V}_{\text {day } 0}\right) \times 100$, where $\mathrm{V}_{\text {dayi }}$ is the spheroid volume at the nth day (day 1, 3, 5 or 7) of treatment, and $V_{\text {day } 0}$ is the spheroid volume prior to treatment.

In vivo tumor-targeting imaging. DiR, a near-infrared fluorescent probe, was encapsulated into PTX Emul and PTX-CH Emul. To investigate the tumor-targeting efficiency of PTX-CH Emul in the MDA-MB-231 tumor-bearing nude mice, PBS, DiR-encapsulated PTX Emul or DiR-encapsulated PTX-CH Emul was injected into the mice via the tail vein at a dose of $200 \mu \mathrm{g}$ of DiR/ $\mathrm{kg}$ body weight. The in vivo imaging was performed at different time points (2, 4, 8, 12 and $24 \mathrm{~h})$ post-injection using the In vivo IVIS spectrum imaging system (Perkin-Elmer, Wellesley, MA, USA). At $24 \mathrm{~h}$ post-injection, the mice were sacrificed and the tumors and other major organs (heart, liver, spleen, lung and kidney) were harvested. The ex vivo imaging of the organs was also carried out.

Maximum tolerated dose (MTD) determination. Healthy female BALB/c nude mice were used to determine the MTD of PTX-CH Emul and Taxol after intravenous administration. Eight groups of 5-8 female BALB/c nude mice received intravenous injections of Taxol (20, 30 or $45 \mathrm{mg} / \mathrm{kg})$, PTX-CH Emul $(30,45,67.5$ or $101.25 \mathrm{mg} / \mathrm{kg}$ ) or saline (as a control) via the tail vein on days 0,4 and 8 . The effects of the treatments were investigated by close observation of survival rates. The MTD was defined as the dose that caused neither death resulting from toxic effects nor significant changes in general clinical signs within 1 week after intravenous administration of the aforementioned formulations.

In vivo antitumor effect. The antitumor efficacy and toxicity profiles of the different PTX formulations were evaluated in a subcutaneous MDA-MB-231 xenograft model in female $\mathrm{BALB} / \mathrm{c}$ nude mice. The treatments commenced when the tumors in the nude mice reached a volume of $100-200 \mathrm{~mm}^{3}$, and this day was designated as day 0 . On day 0 , the mice were randomly divided into 3 groups of 8-9 mice each. The mice were intravenously administered PBS, Taxol $(20 \mathrm{mg} / \mathrm{kg})$ or PTX-CH Emul $(45 \mathrm{mg} / \mathrm{kg})$, respectively. The treatments were conducted every 4 days for a total of 3 doses. Tumor size was measured with a digital caliper every 3 days. Tumor volume was calculated by the formula of $\left(\mathrm{LxW}^{2}\right) / 2$, where $\mathrm{L}$ is the longest tumor dimension $(\mathrm{mm})$ and $\mathrm{W}$ is the shortest tumor dimension $(\mathrm{mm})$. The time required for a tumor to double in volume was calculated based on the initial tumor volume at the beginning of the treatment. Tumor recurrence was defined as the first observation of increased tumor size following tumor regression. The toxicity after treatment was monitored by observing behavior and measuring body weight every 3 days.

Statistical analysis. All data subjected to statistical analysis were obtained from at least 3 parallel experiments, and the results are expressed as mean \pm standard deviation (SD). Tumor doubling time and time to tumor recurrence were analyzed by Kaplan-Meier analysis, according to GraphPad Prism version 5.01 for Windows (GraphPad ${ }^{\circledR}$, Inc., Software, San Diego, CA, USA). Statistical analysis was performed by Student's t-test for two groups, and one way ANOVA for multiple groups. A value of $\mathrm{P}<0.05$ was considered statistically significant.

\section{Results}

Characterization of PTX-CH Emul. The characteristics of PTX-CH Emul were previously investigated (22). PTX-CH Emul, which is composed of a paclitaxel-cholesterol complex surrounded by a phospholipid monolayer, resembles the lipid structure of native LDL (Fig. 1A). The resulting emulsion had an encapsulation efficiency of $97.3 \%$ and a mean particle size of $150 \mathrm{~nm}$ (Fig. 1B). Moreover, the formulation survived autoclaving at $115^{\circ} \mathrm{C}$ for $30 \mathrm{~min}$ and remained stable for at least 12 months at $6^{\circ} \mathrm{C}$. PTX-CH Emul was also substantially better tolerated than the corresponding dosages of Taxol in guinea pigs, as no evident hypersensitivity reaction was observed. These results demonstrated that PTX-CH Emul has excellent potential for industrial-scale production and clinical application.

CLSM is a useful tool in the investigation of the microstructure of emulsions $(26,27)$. In our study, the distribution 
A

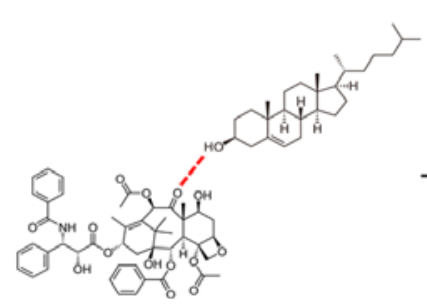

Paclitaxel + Cholesterol

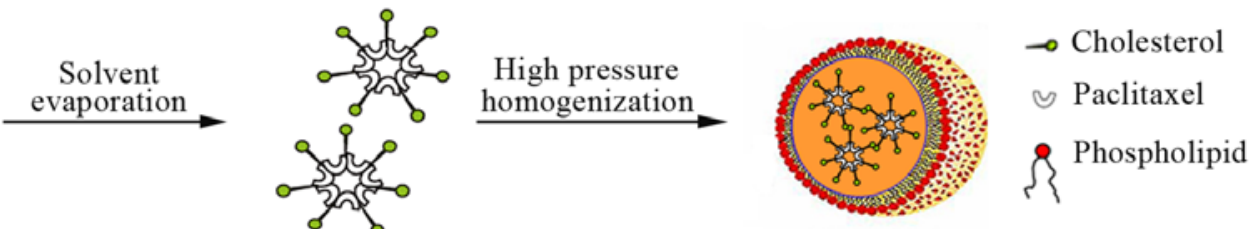

Paclitaxel-cholesterol complex
Paclitaxel-cholesterol emulsion
B

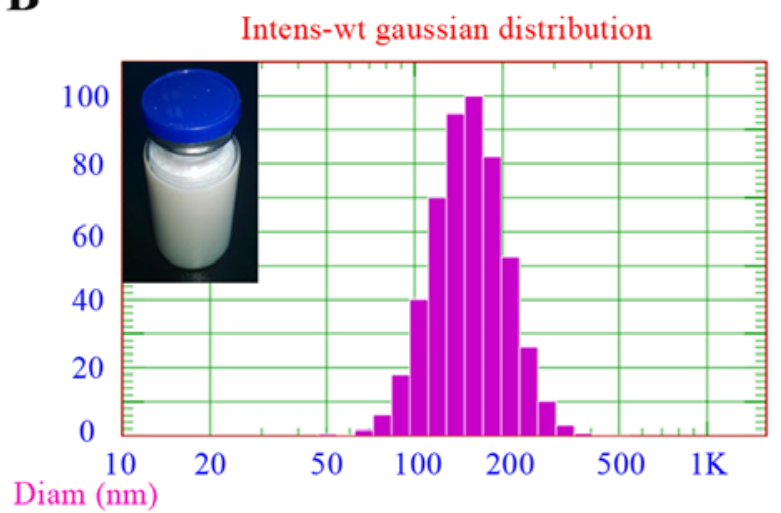

C

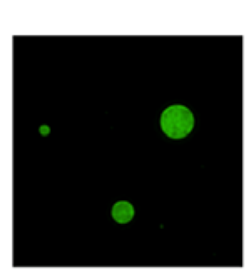

$\mathbf{E}$

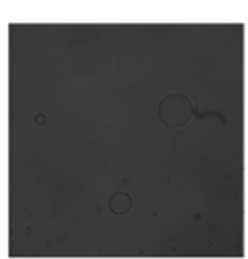

D

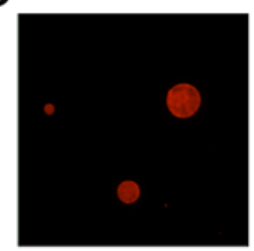

$\mathbf{F}$

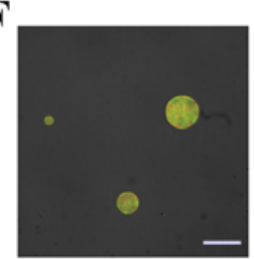

Figure 1. (A) Schematic representation of the paclitaxel-cholesterol emulsion (PTX-CH Emul). (B) Particle size and size distribution determined by the PSS NICOMP Particle Size System (Port Richey, FL, USA). Confocal laser scanning micrographs of emulsion recorded with (C) a green fluorescent dye (fluorescein) labelling the fluorescein-cholesterol complex, (D) a red fluorescent dye (Nile red) labelling the oil, (E) no excitation light and (F) the merged image. The bar is $25 \mu \mathrm{m}$.

of the fluorescein-cholesterol complex and oil in the emulsion was determined by CLSM. Oil was labeled with a lipid probe, Nile red, a hydrophobic fluorescent marker with special fluorescent properties. Nile red emitted red light and was excited to visualize the distribution of the oil core in the emulsion. Fluorescein emitted green light was excited to visualize the distribution of the fluorescein-cholesterol complex in the emulsion. Yellow light was observed when Nile red and fluorescein were both excited and localized in the same region. The micrographs revealed a uniform distribution of Nile red and fluorescein in the emulsion. The green and red regions completely overlapped, indicating that the fluorescein-cholesterol complex and oil phase were evenly distributed throughout the emulsion, without phase separation (Fig. 1C-F).

In vitro cytotoxicity assay. The cytotoxicity of Blank Emul, PTX-CH Emul, PTX Emul and Taxol was evaluated using MDA-MB-231 cells. PTX decreased cell viability in a concentration-dependent manner in the concentration range of $0.001-0.05 \mu \mathrm{g} / \mathrm{ml}$ (Fig. 2A). The cell-survival rate in the group treated with PTX-CH Emul was significantly lower than that in the groups treated with PTX Emul and Taxol after $72 \mathrm{~h}$, demonstrating that PTX-CH Emul had better anticancer efficacy than PTX Emul and Taxol.

An important parameter for quantitatively evaluating the in vitro cytotoxicity of an anticancer drug is the $\mathrm{IC}_{50}$ or $\mathrm{IC}_{70}$, which represents the drug concentration needed to kill $50 \%$ or $70 \%$ of the cancer cells at a designated time $(28,29)$. Fig. $2 \mathrm{~B}$ and $\mathrm{C}$ shows the $\mathrm{IC}_{50}$ and $\mathrm{IC}_{70}$ values of PTX-CH
Emul, PTX Emul and Taxol in the MDA-MB-231 cells after a 72-h incubation, respectively. Among the different PTX formulations, PTX-CH Emul had the lowest $\mathrm{IC}_{50}$ value $(2.13 \pm 0.28 \mathrm{ng} / \mathrm{ml})$, followed by PTX Emul $(3.02 \pm 0.19 \mathrm{ng} / \mathrm{ml}$, $\mathrm{P}<0.05)$ and Taxol $(4.22 \pm 0.52 \mathrm{ng} / \mathrm{ml}, \mathrm{P}<0.001)$. If $\mathrm{IC}_{70}$ value was used, the differences were more pronounced since the $\mathrm{IC}_{70}$ value of PTX-CH Emul $(4.69 \pm 0.36 \mathrm{ng} / \mathrm{ml})$ was significantly lower than that of all the other formulations, only $\sim 1.5$ - and 2.4-fold lower than that of PTX Emul $(7.14 \pm 0.63 \mathrm{ng} / \mathrm{ml}$, $\mathrm{P}<0.01)$ and Taxol $(11.07 \pm 0.93 \mathrm{ng} / \mathrm{ml}, \mathrm{P}<0.001)$ respectively. These results demonstrated that PTX-CH Emul exhibited superior cytotoxicity as compared to PTX Emul and Taxol. Additionally, there was no cellular cytotoxicity caused by the Blank Emul at the investigated concentrations, eliminating the possibility that the lipid emulsions themselves were responsible for the cytotoxicity.

In vitro cellular uptake. The cellular uptake profiles of FL-DMSO, FL emulsion and FL-CH emulsion in the MDA-MB-231 cells were assessed qualitatively using CLSM. As shown in Fig. 3, FL-CH emulsion exhibited significantly greater uptake in comparison with FL-DMSO and FL emulsion. This result demonstrated that emulsion loaded with a fluorescein-cholesterol complex significantly facilitated the uptake of fluorescein by the MDA-MB-231 cells.

Growth inhibition of 3D breast tumor spheroids. In vitro 3D tumor spheroids provide an important link between monolayer cell cultures and animal models for evaluating drug delivery 
A
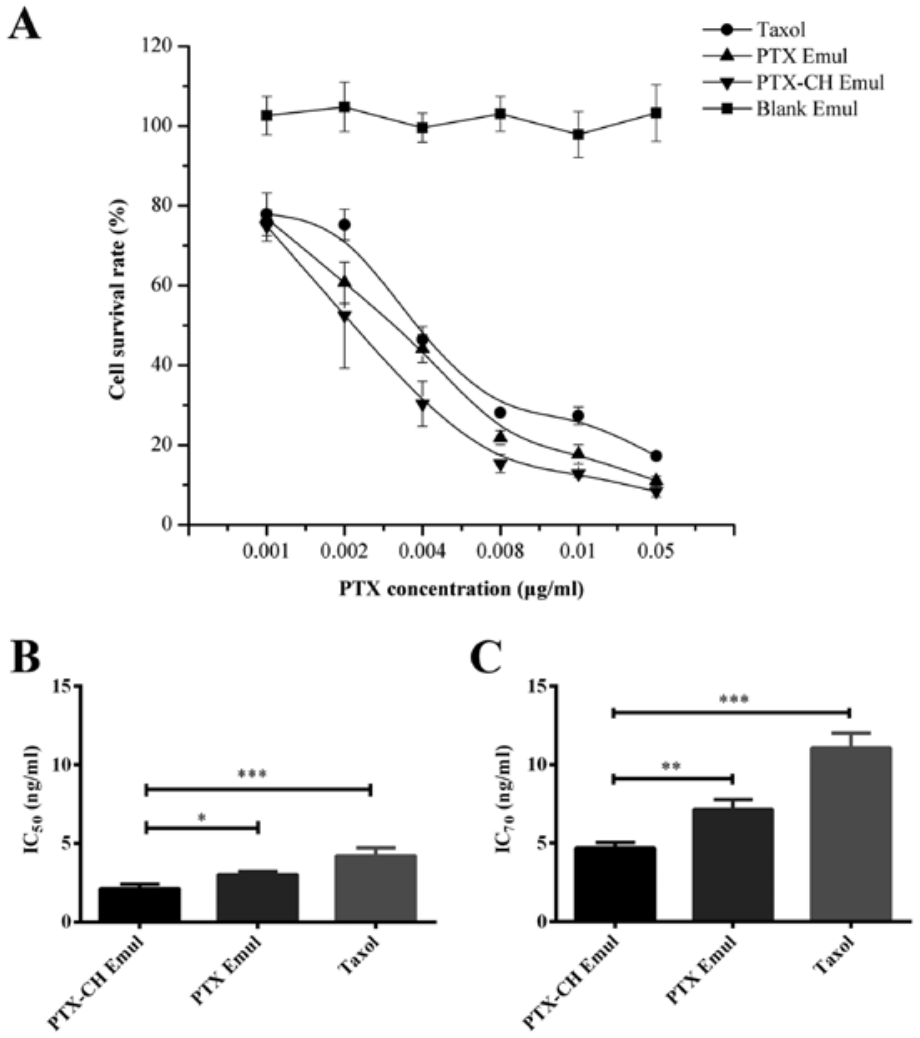

Figure 2. (A) In vitro cytotoxicity studies of Blank Emul, PTX-CH Emul, PTX Emul and Taxol in MDA-MB-231 cells at $72 \mathrm{~h}$. (B) The IC $\mathrm{C}_{50}$ values of PTX-CH Emul, PTX Emul and Taxol. (C) The $\mathrm{IC}_{70}$ values of PTX-CH Emul, PTX Emul and Taxol. Each value represents the mean \pm SD $(n=3)$. " P<0.05, ** P<0.01 and ${ }_{* * * *} \mathrm{P}<0.001$. PTX Emul, paclitaxel emulsion; PTX-CH Emul, paclitaxel-cholesterol complex emulsion.

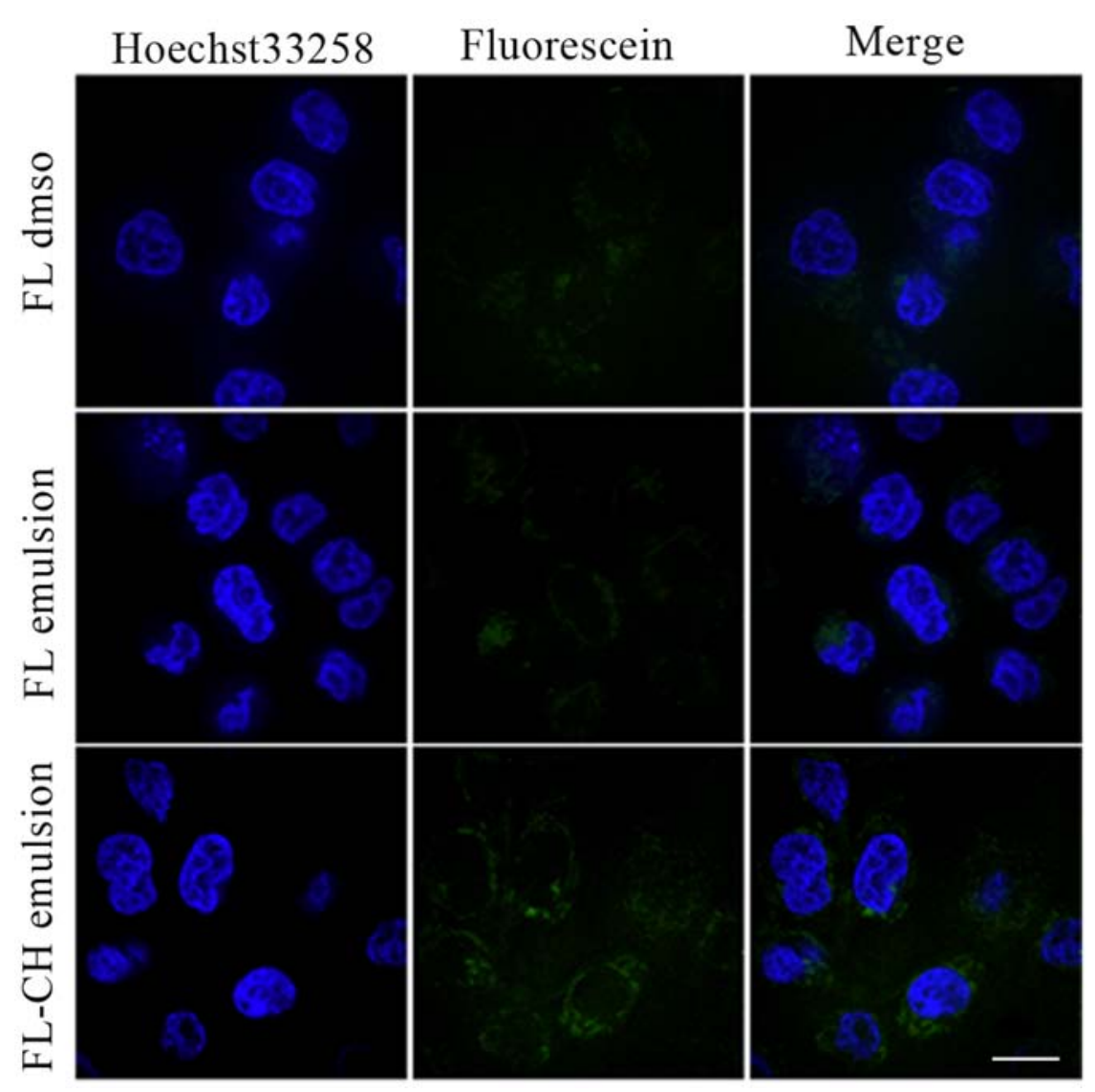

Figure 3. Confocal laser scanning micrographs of MDA-MB-231 cells incubated with the FL-DMSO, FL emulsion and FL-CH emulsion diluted in culture medium with $10 \% \mathrm{FBS}$ at $37^{\circ} \mathrm{C}$. Scale bar, $14 \mu \mathrm{m}$. FL-DMSO, fluorescein-DMSO solution; FL emulsion, emuslion loaded with fluorescein; FL-CH emulsion, emulsion loaded with fluorescein-cholesterol complex. 

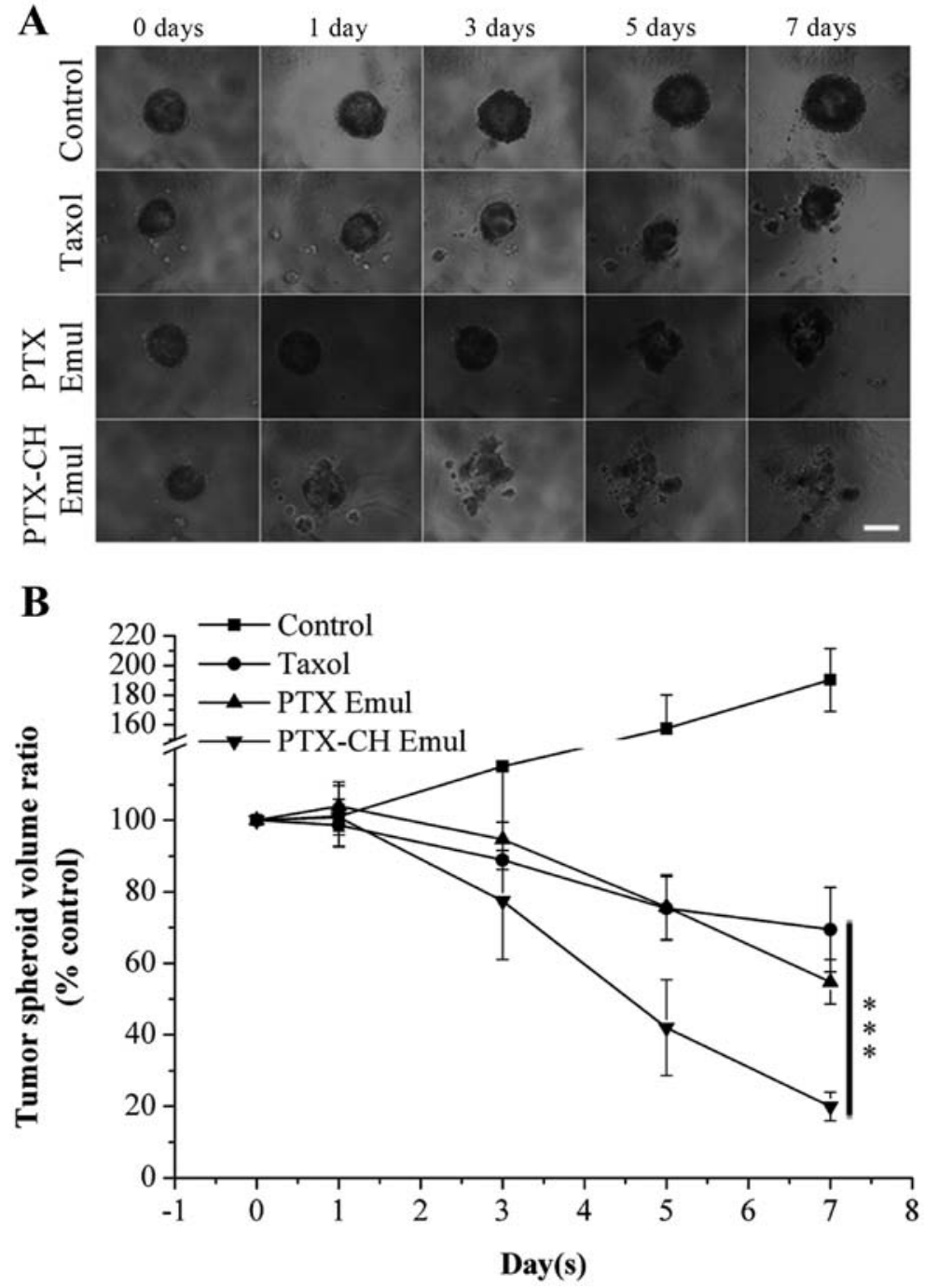

Figure 4. (A) Inhibition of MDA-MB-231 tumor spheroid growth was evaluated following the treatment with PTX-CH Emul, PTX Emul and Taxol, respectively, at a paclitaxel concentration of $0.5 \mu \mathrm{g} / \mathrm{ml}$ with those treated with culture medium as the blank control. The images were obtained at $0,1,3,5$ and 7 days under inverted microscope. Scale bar, $300 \mu \mathrm{m}$. (B) Changes in MDA-MB-231 tumor spheroid volumes (\%) after treatment with various paclitaxel formulations (PTX-CH Emul, PTX Emul and Taxol) and culture medium. Each value represents the mean $\pm \mathrm{SD}(\mathrm{n}=6) .{ }^{* * *} \mathrm{P}<0.001$. PTX Emul, paclitaxel emulsion; PTX-CH Emul, paclitaxel-cholesterol complex emulsion.

efficiency (30,31). 3D tumor spheroids generated by the liquid overlay technique contain aggregates of cells in close contact and an organized extracellular matrix consisting of fibronectin, laminin and collagen, which is similar to the extracellular matrix of tumors in vivo (32). Therefore, 3D breast tumor spheroids were used to evaluate the inhibitory effect of PTX-CH Emul on cell growth. 3D tumor spheroid growth was evaluated following the treatment of cells with culture medium containing PTX-CH Emul, PTX Emul or Taxol. In regular cell culture medium, 3D tumor spheroids of MDA-MB-231 cells grew rapidly and became more compact with the increase in culture time. However, in the presence of PTX Emul or Taxol, the tumor spheroids stopped growing or even became smaller. Distinctly, treatment of cells with PTX-CH Emul resulted in shrinkage of the spheroids, with the concomitant loss of 3D structures due to the dissociation of some cells from the spheroids, indicating that the PTX-CH Emul effectively inhibited tumor cell proliferation (Fig. 4A). After 7 days of treatment, the tumor spheroid volume ratios were $190.2 \pm 21.2 \%$ for the control group, $69.5 \pm 11.8 \%$ for the Taxol group, $54.8 \pm 6.2 \%$ for the PTX Emul group and $19.9 \pm 4.0 \%$ for the PTX-CH Emul group (Fig. 4B). All things considered, PTX-CH Emul exhibited much stronger inhibitory effects on tumor spheroids in comparison with Taxol and PTX Emul.

In vivo tumor-targeting imaging. The tumor-targeting efficiency of PTX-CH Emul was evaluated in nude mice bearing MDA-MB-231 tumors. After the DiR-labeled emulsions were injected through the tail vein, the time-dependent biodistribution of different emulsions was monitored using non-invasive NIR fluorescence imaging in live animals. As shown in Fig. 5A, at all time points post-injection, the tumor fluorescence in DiR-PTX-CH Emul-treated mice was higher than that in the DiR-PTX Emul-treated mice at equivalent doses of DiR. Twenty-four hours after injection, 3D reconstruction of DiR-PTX-CH Emul-treated mice was performed. As shown in Fig. 5C, the fluorescence was primarily found at tumor sites, indicating that the DiR-PTX-CH Emul may serve as an effective tumor-targeting vehicle by which to deliver chemotherapeutic drugs to tumor tissues. Additionally, 
A

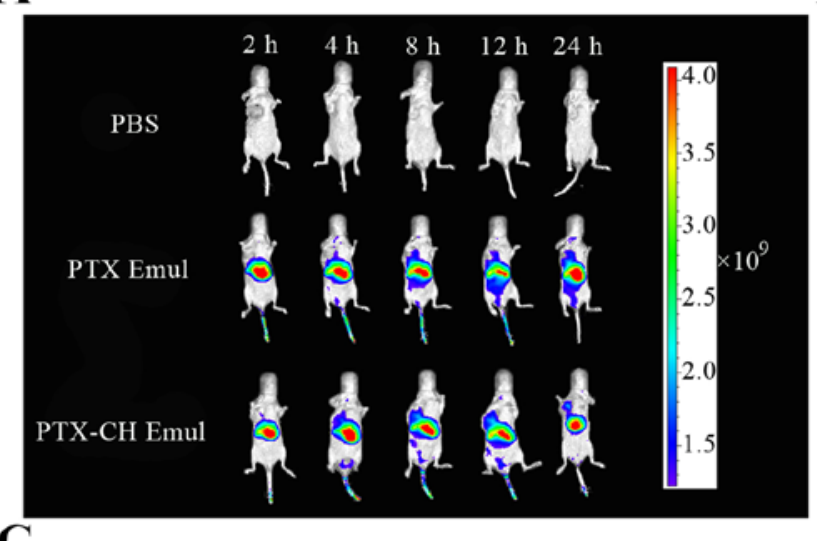

B

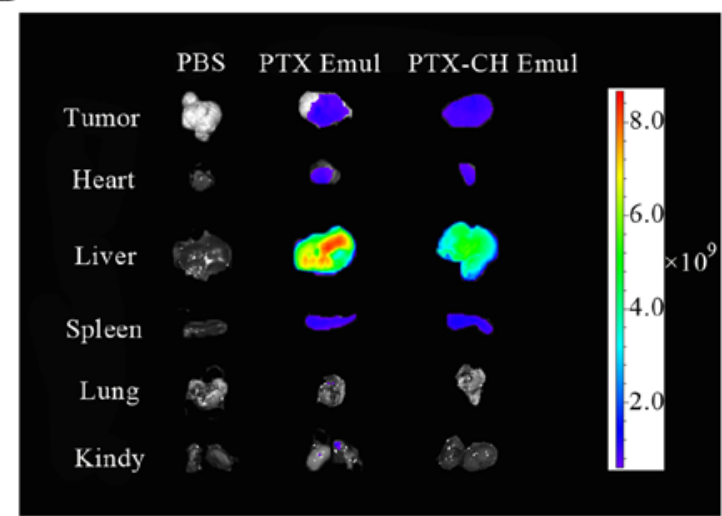

C

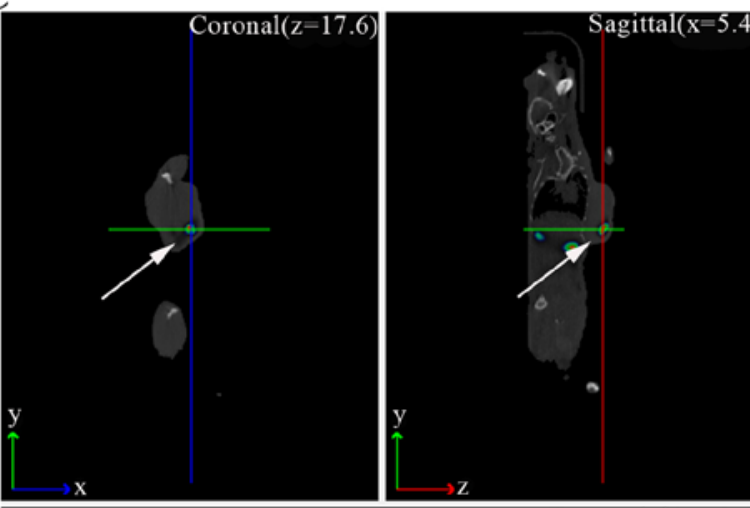

ransaxial $(y=-0.4$
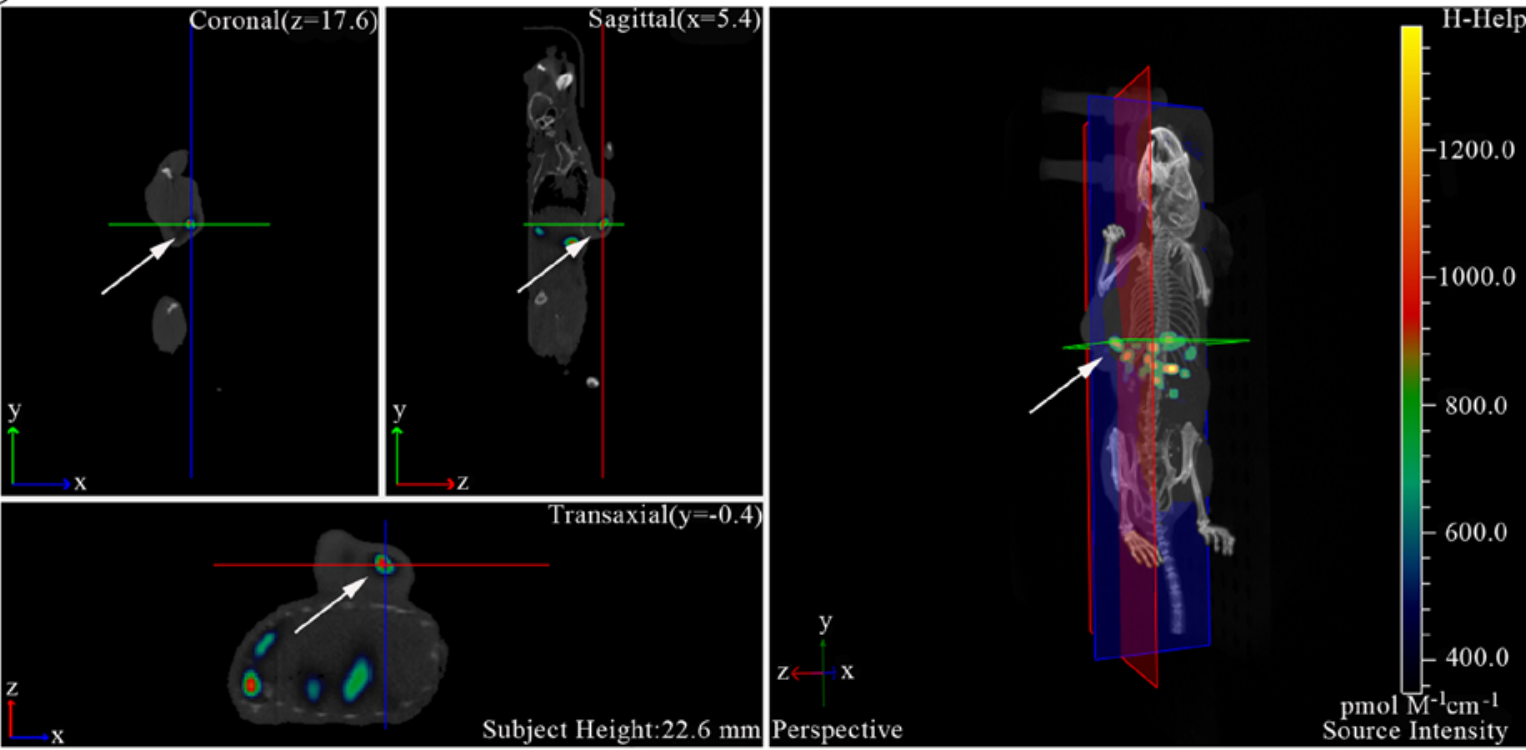

Figure 5. (A) In vivo fluorescence images of nude mice bearing MDA-MB-231 tumor cells after tail vein injection of PBS, DiR-PTX Emul and DiR-PTX-CH Emul. Images were captured at predetermined time intervals. (B) Ex vivo fluorescence images of excised organs and tumors at $24 \mathrm{~h}$ post-injection of PBS, DiR-PTX Emul and DiR-PTX-CH Emul. (C) 3D reconstruction of in vivo imaging of DiR-PTX-CH Emul-treated mice. PBS, phosphate-buffered saline; PTX Emul, paclitaxel emulsion; PTX-CH Emul, paclitaxel-cholesterol complex emulsion.

the ex vivo fluorescent image from the excised tumors also clearly showed higher fluorescence from the DiR-PTX-CH Emul-treated group over that from the DiR-PTX Emul-treated one (Fig. 5B), which was further evidence of the higher tumortargeting efficiency of PTX-CH Emul. All these findings indicated that PTX-CH Emul enabled specific tumor-targeting efficiency, which could contribute to the selective increase in the therapeutic efficiency.

Maximum tolerated dose. The MTD of Taxol in healthy $\mathrm{BALB} / \mathrm{c}$ nude mice is $20 \mathrm{mg} / \mathrm{kg}$, consistent with the previously reported results (33). PTX-CH Emul was significantly less toxic than Taxol, and the MTD of PTX-CH Emul was 2.25 -fold higher than that of Taxol $(45 \mathrm{mg} / \mathrm{kg}$ vs. $20 \mathrm{mg} / \mathrm{kg}$, respectively). A previous study reported that the MTD of Abraxane ${ }^{\circledR}$ is 2.24 -fold higher than that of Taxol $(30 \mathrm{mg} / \mathrm{kg}$ vs. $13.4 \mathrm{mg} / \mathrm{kg}$, respectively) (34), which is essentially the same as that reported here. Based on these results, MTD values of $45 \mathrm{mg} / \mathrm{kg}$ and $20 \mathrm{mg} / \mathrm{kg}$ were chosen for PTX-CH Emul and Taxol, respectively, for the in vivo antitumor efficiency study.
In vivo antitumor efficiency. Both Taxol and PTX-CH Emul significantly inhibited tumor growth in comparison with the control group (Fig. 6A). At the MTDs of PTX-CH Emul and Taxol (45 and $20 \mathrm{mg} / \mathrm{kg}$, respectively), the proportion of tumor-free survivors in the PTX-CH Emul group (7 of 9) was higher than that in the Taxol group (0 of 8) (Table I). Equally as important, PTX-CH Emul showed superior antitumor activity to Taxol as measured by median time to tumor recurrence [ $>79$ days vs. 32 days $(\mathrm{P}=0.0001)]$ (Fig. $6 \mathrm{~B}$ and Table I), tumor doubling time $[>79$ days vs. 7.5 days $(\mathrm{P}<0.0001)]$ (Fig. 6C and Table I), tumor volume $(\mathrm{P}<0.01)$ (Fig. 6A) and tumor weight $(\mathrm{P}<0.001)$ (Fig. 6D). These results demonstrated that PTX-CH Emul possessed higher antitumor efficacy as compared with Taxol at the MTDs.

Toxicity was assessed by direct observation of animal behavior and body weight. Mice treated with $20 \mathrm{mg} / \mathrm{kg}$ Taxol showed reduced overall activity, which was likely a sign of a hypersensitivity reaction to the diluent. In contrast, the mice treated with PTX-CH Emul tolerated the regimens well. As shown in Fig. 6E, there was no significant difference in body weight loss between mice treated with PTX-CH Emul and 

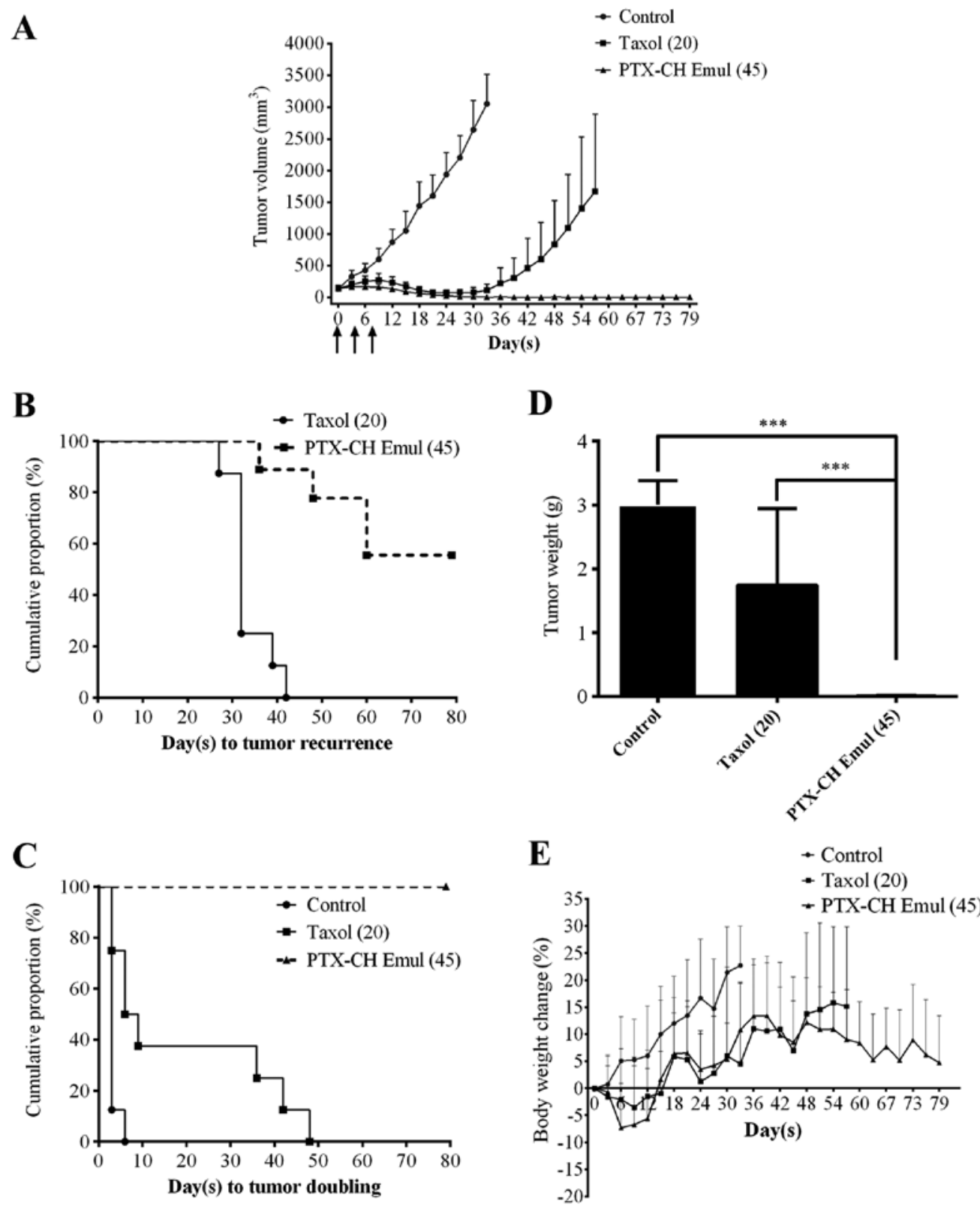

Figure 6. (A) In vivo tumor growth inhibition, (B) Kaplan-Meier analysis of tumor recurrence, (C) Kaplan-Meier analysis of tumor doubling time, (D) tumor weight and (E) body weight changes of MDA-MB-231 tumor-bearing nude mice after the intravenous treatment of various paclitaxel formulations. ${ }^{* * *} \mathrm{P}<0.001$.

Table I. Antitumor activity of PTX-CH Emul and Taxol at the MTDs

\begin{tabular}{lccccc}
\hline Xenograft treatment & Tumor-free survivors & Recurrence (days) $^{\mathrm{a}}$ & P-value $^{\mathrm{c}}$ & Tumor doubling (days) $^{\mathrm{b}}$ & P-value $^{\mathrm{c}}$ \\
\hline Control & $0 / 8$ & - & - & 3.0 & $<0.0001$ \\
Taxol $^{\otimes}(20 \mathrm{mg} / \mathrm{kg})$ & $0 / 8$ & 32 & 0.0001 & 7.5 & $<0.0001$ \\
PTX-CH Emul $(45 \mathrm{mg} / \mathrm{kg})$ & $7 / 9$ & $>79$ & - & $>79.0$ & -
\end{tabular}

PTX-CH Emul, paclitaxel-cholesterol emulsion. ${ }^{a}$ The first observation of increased tumor size following tumor regression. ${ }^{\mathrm{b}}$ Time required for tumors to double in volume from the initial dosing day. ${ }^{\circ}$ Compared with PTX-CH Emul at $45 \mathrm{mg} / \mathrm{kg}$.

Taxol at the MTDs. Mice had reduced body weight initially after treatment with the aforementioned formulations but gradually began to grow normally after they adapted to the challenges.

\section{Discussion}

It has been previously reported that an artificial emulsion resembling the lipid structure of LDL, designated as LDE, 
has the ability to bind to LDL receptors. Upon intravenous administration, LDE picks up circulating apolipoproteins (apo), such as apo E, from the native lipoproteins. The LDL receptor recognizes and binds apo $\mathrm{E}$ with high affinity, thereby coupling the LDE particles to the receptor and enabling internalization of LDE into the cytoplasm $(13,35)$. Accumulated evidence has demonstrated that many tumor cells have high LDL requirements and LDL receptors are overexpressed in many tumor cells due to the rapid growth of these malignant cells and their requirement for structural lipids (17-19). In a previous study, Rodrigues et al reported that PTX can be associated at high rates with LDE (LDE-PTX), which is developed for the targeted treatment of lung carcinoma. However, LDE-PTX is stable only for 8 days at $4^{\circ} \mathrm{C}$ and tends to dissociate in the bloodstream after injection into mice $(13,14)$. Long-term stability is one of the essential elements for druggability (6). The instability of LDE-PTX makes it less promising for clinical evaluation. In our previous study, we successfully developed a PTX-CH Emul resembling the LDL lipid structure, which is composed of a paclitaxel-cholesterol complex surrounded by a phospholipid monolayer (22). Compared with the mixture of PTX and cholesteryl ester in LDE-PTX, the paclitaxel-cholesterol complex connected by intermolecular hydrogen bonds between PTX and cholesterol is used as the drug carrier of the PTX-CH Emul. The paclitaxel-cholesterol complex not only dramatically improves the lipophilicity of PTX, but can also function as the component of LDL-cholesterol. The resulting emulsion exhibits an ideal particle size, high drug loading capability, high drug encapsulation efficiency and excellent stability $\left(12\right.$ months at $\left.6^{\circ} \mathrm{C}\right)$. The long-term stability of PTX-CH Emul is of critical importance not only for therapeutic purposes but also for the development of the formulation since stability is of major concern to the pharmaceutical industry.

Since LDL receptors have been identified to be overexpressed in breast cancer cells and the LDL receptor mRNA level in TNBC cells is more abundant than the level in non-TNBC cells (19), MDA-MB-231, a human TNBC cell line was used in this study. TNBC is an aggressive, heterogeneous subclass of breast cancer typically of basal origin, accounting for $15-20 \%$ of all invasive breast cancers. Unfortunately, TNBC presents a difficult clinical challenge as it is ultimately refractory to chemotherapy, and displays a shorter median time to relapse and death than other subtypes of breast cancer $(36,37)$. Therefore, treatment of the MDA-MB-231 xenograft tumor model with PTX-CH Emul provides a stringent assessment of the potential clinical utility of this formulation.

In order to evaluate and compare the in vitro antitumor efficacy of PTX-CH Emul and Taxol ${ }^{\circledR}, 2 \mathrm{D}$ monolayer tumor cells and 3D multicellular tumor spheroids were used as the models. Our in vitro cytotoxicity study of 2D monolayer cells demonstrated that no cytotoxicity of Blank Emul was observed in the MDA-MB-231 cells. When lipid emulsions were loaded with the paclitaxel-cholesterol complex, they exhibited superior cytotoxicity compared to Taxol as reflected by the corresponding $\mathrm{IC}_{50}$ and $\mathrm{IC}_{70}$ values. Previous studies reported that the LDE, which is basically composed of a cholesteryl ester core surrounded by a phospholipid monolayer, could bind to LDL receptors $(13,35)$. Therefore, the PTX-CH Emul reported here, which is composed of a paclitaxel-cholesterol complex core surrounded by a phospholipid monolayer, may serve as a tumor-targeting vehicle for PTX directed against MDA-MB-231 cells with abundant LDL receptors thus greatly improving the in vitro cytotoxicity of PTX. In order to verify this hypothesis, a comparative in vitro experiment was performed to test the antitumor effects of PTX-CH Emul and PTX Emul. In contrast to PTX-CH Emul, PTX Emul, which is basically composed of a paclitaxel core, does not resemble the lipid structure of LDL. As reflected by the corresponding $\mathrm{IC}_{50}$ and $\mathrm{IC}_{70}$ values, PTX-CH Emul showed better cytotoxicity compared to PTX Emul. Additionally, the result of in vitro cellular uptake demonstrated that FL-CH emulsion exhibited significantly greater uptake in MDA-MB-231 cells as compared to FL solution and FL emulsion. Therefore, we surmise that the superior cytotoxicity of PTX-CH Emul over other tested PTX formulations may be attributed to the tumortargeting effect of PTX-CH Emul. However, whether the tumor-targeting effect of PTX-CH Emul is mediated by LDL receptors still remains to be confirmed in our future studies.

Compared with the conventional 2D monolayer model, the 3D tumor spheroid model has a closer resemblance to cells growing in the in vivo tissue microenvironment by mimicking many of the physiological characteristics of the native tumor environment, including complex multicellular architecture, barriers to mass transport, and extracellular matrix deposition. More importantly, 3D tumor spheroids are more chemo-resistant compared to monolayer cells and thus could serve as excellent models for evaluating the efficiency of drug delivery systems $(38,39)$. Similar to the results of in vitro cytotoxicity in the 2D monolayer tumor cells, PTX-CH Emul exhibited stronger and faster inhibitory effects on 3D tumor spheroids as compared with PTX Emul and Taxol. All in all, the enhanced 3D multicellular tumor spheroid growth inhibition of PTX-CH Emul may be attributed to the superior cytotoxicity in the 2D monolayer MDA-MB-231 cells and the capability of LDL receptors to facilitate tumor penetration and tumor accumulation of PTX-CH Emul in 3D tumor spheroids.

In order to evaluate tumor-targeting efficiency and monitor the real-time biodistribution of PTX-CH Emul in vivo, non-invasive near infrared fluorescence optical imaging technology was employed. Compared to the conventional PTX Emul, PTX-CH Emul primarily accumulated in tumors in the mice-bearing MDA-MB-231 xenografts after intravenous administration, possibly as a result of both enhanced permeability and retention (EPR) effects and LDL receptor-mediated tumor-targeting effects.

To evaluate the in vivo antitumor efficiency of PTX-CH Emul on MDA-MB-231 tumor-bearing mice, PBS, PTX-CH Emul $(45 \mathrm{mg} / \mathrm{kg})$ or Taxol $(20 \mathrm{mg} / \mathrm{kg})$ was intravenously injected into mice. As chemotherapy is generally administered at the highest tolerated dose, the comparison of MTDs, rather than equal doses, is considered to be more clinically relevant (34). The MTD of PTX-CH Emul was 2.25-fold of that of Taxol (45 vs. $20 \mathrm{mg} / \mathrm{kg}$, respectively), which was basically the same as the fold ratio of the MTD of Abraxane to that of Taxol (30 vs. $13.4 \mathrm{mg} / \mathrm{kg}, 2.24$-fold) as previously reported (34). The result of MTD indicated that PTX-CH Emul exhibited better safety profiles in vivo than Taxol. At the MTDs, PTX-CH Emul exhibited higher antitumor activity in the TNBC models as compared to Taxol. There are several possible explanations 
for the enhanced antitumor efficacy of PTX-CH Emul after intravenous injection. Firstly, PTX-CH Emul has a high tumortargeting ability, thus resulting in a higher tumor accumulation as compared with PTX Emul. Secondly, the much stronger and faster inhibitory effects of PTX-CH Emul on 2D monolayer cells and 3D multicellular tumor spheroids should contribute to the enhanced antitumor efficacy of PTX-CH Emul.

In conclusion, the results of the present study suggest that the lipid emulsion based on the paclitaxel-cholesterol complex reported here has great clinical potential for the treatment of breast tumors.

\section{Acknowledgments}

This study was financially supported by the National Megaproject for Innovative Drugs (no. 2012ZX09301002-001) and Beijing Municipal Science \& Technology Commission Preclinical Research Projects (no. 500101009).

\section{References}

1. Rowinsky EK and Donehower RC: Paclitaxel (Taxol). N Engl J Med 332: 1004-1014, 1995.

2. Rowinsky EK, Cazenave LA and Donehower RC: Taxol: a novel investigational antimicrotubule agent. J Natl Cancer Inst 82: 1247-1259, 1990.

3. Weiss RB, Donehower RC, Wiernik PH, Ohnuma T, Gralla RJ, Trump DL, Baker JR Jr, Van Echo DA, Von Hoff DD and Leyland-Jones B: Hypersensitivity reactions from Taxol. J Clin Oncol 8: 1263-1268, 1990.

4. Singla AK, Garg A and Aggarwal D: Paclitaxel and its formulations. Int J Pharm 235: 179-192, 2002.

5. Koudelka S and Turanek J: Liposomal paclitaxel formulations. Journal Control Release 163: 322-334, 2012.

6. Jing X, Deng L, Gao B, Xiao L, Zhang Y, Ke X, Lian J, Zhao Q, Ma L, Yao J, et al: A novel polyethylene glycol mediated lipid nanoemulsion as drug delivery carrier for paclitaxel. Nanomedicine 10: 371-380, 2014.

7. Zhang $Z$ and Feng SS: The drug encapsulation efficiency, in vitro drug release, cellular uptake and cytotoxicity of paclitaxel-loaded poly(lactide)-tocopheryl polyethylene glycol succinate nanoparticles. Biomaterials 27: 4025-4033, 2006.

8. Huh KM, Min HS, Lee SC, Lee HJ, Kim S and Park K: A new hydrotropic block copolymer micelle system for aqueous solubilization of paclitaxel. J Control Release 126: 122-129, 2008.

9. Joshi MD and Muller RH: Lipid nanoparticles for parenteral delivery of actives. Eur J Pharm Biopharm 71: 161-172, 2009.

10. Lammers T, Kiessling F, Hennink WE and Storm G: Drug targeting to tumors: principles, pitfalls and (pre-) clinical progress. J Control Release 161: 175-187, 2012.

11. Constantinides PP, Tustian A and Kessler DR: Tocol emulsions for drug solubilization and parenteral delivery. Adv Drug Deliv Rev 56: 1243-1255, 2004.

12. Collins-Gold L, Lyons $\mathrm{R}$ and Bartholow L: Parenteral emulsions for drug delivery. Adv Drug Deliv Rev 5: 189-208, 1990.

13. Rodrigues DG, Covolan CC, Coradi ST, Barboza R and Maranhão RC: Use of a cholesterol-rich emulsion that binds to low-density lipoprotein receptors as a vehicle for paclitaxel. J Pharm Pharmacol 54: 765-772, 2002.

14. Rodrigues DG, Maria DA, Fernandes DC, Valduga CJ, Couto RD, Ibañez OC and Maranhão RC: Improvement of paclitaxel therapeutic index by derivatization and association to a cholesterol-rich microemulsion: in vitro and in vivo studies. Cancer Chemother Pharmacol 55: 565-576, 2005.

15. Pires LA, Hegg R, Valduga CJ, Graziani SR, Rodrigues DG and Maranhão RC: Use of cholesterol-rich nanoparticles that bind to lipoprotein receptors as a vehicle to paclitaxel in the treatment of breast cancer: pharmacokinetics, tumor uptake and a pilot clinical study. Cancer Chemother Pharmacol 63: 281-287, 2009
16. Teixeira RS, Valduga CJ, Benvenutti LA, Schreier S and Maranhão RC: Delivery of daunorubicin to cancer cells with decreased toxicity by association with a lipidic nanoemulsion that binds to LDL receptors. J Pharm Pharmacol 60: 1287-1295, 2008.

17. Vitols S, Peterson C, Larsson O, Holm P and Aberg B: Elevated uptake of low density lipoproteins by human lung cancer tissue in vivo. Cancer Res 52: 6244-6247, 1992.

18. Maletínská L, Blakely EA, Bjornstad KA, Deen DF, Knoff LJ and Forte TM: Human glioblastoma cell lines: levels of low-density lipoprotein receptor and low-density lipoprotein receptor-related protein. Cancer Res 60: 2300-2303, 2000.

19. Stranzl A, Schmidt H, Winkler R and Kostner GM: Low-density lipoprotein receptor mRNA in human breast cancer cells: influence by PKC modulators. Breast Cancer Res Treat 42: 195-205, 1997.

20. Xia X, Song X, Xu J, He J, Peng J, Zhang X, Jin D, Abliz Z and Liu Y: Development of a validated LC-APCI-MS/MS method to study the plasma and tumor distribution of CHO-PTX intravenous lipid emulsion. J Pharm Biomed Anal 117: 532-543, 2016.

21. Stevens PJ, Sekido M and Lee RJ: A folate receptor-targeted lipid nanoparticle formulation for a lipophilic paclitaxel prodrug. Pharm Res 21: 2153-2157, 2004.

22. Xia XJ, Guo RF, Liu YL, Zhang PX, Zhou CP, Jin DJ and Wang RY: Formulation, characterization and hypersensitivity evaluation of an intravenous emulsion loaded with a paclitaxel-cholesterol complex. Chem Pharm Bull (Tokyo) 59: 321-326, 2011.

23. Torrisi R, Balduzzi A, Ghisini R, Rocca A, Bottiglieri L, Giovanardi F, Veronesi P, Luini A, Orlando L, Viale G, et al: Tailored preoperative treatment of locally advanced triple negative (hormone receptor negative and HER 2 negative) breast cancer with epirubicin, cisplatin, and infusional fluorouracil followed by weekly paclitaxel. Cancer Chemother Pharmacol 62: 667-672, 2008

24. Wang Q, Gong T, Sun X and Zhang Z: Structural characterization of novel phospholipid lipid nanoparticles for controlled drug delivery. Colloids Surf B Biointerfaces 84: 406-412, 2011.

25. Zhang R, Luo K, Yang J, Sima M, Sun Y, Janát-Amsbury MM and Kopeček J: Synthesis and evaluation of a backbone biodegradable multiblock HPMA copolymer nanocarrier for the systemic delivery of paclitaxel. J Control Release 166: 66-74, 2013.

26. Xiao K, Li Y, Lee JS, Gonik AM, Dong T, Fung G, Sanchez E, Xing L, Cheng HR, Luo J, et al: 'OA02' peptide facilitates the precise targeting of paclitaxel-loaded micellar nanoparticles to ovarian cancer in vivo. Cancer Res 72: 2100-2110, 2012.

27. Nagelkerke A, Bussink J, Sweep FC and Span PN: Generation of multicellular tumor spheroids of breast cancer cells: how to go three-dimensional. Anal Biochem 437: 17-19, 2013.

28. Bonneau C, Rouzier R, Geyl C, Cortez A, Castela M, Lis R, Daraï $\mathrm{E}$ and Touboul C: Predictive markers of chemoresistance in advanced stages epithelial ovarian carcinoma. Gynecol Oncol 136: 112-120, 2015

29. Bernabeu E, Helguera G, Legaspi MJ, Gonzalez L, Hocht C, Taira C and Chiappetta DA: Paclitaxel-loaded PCL-TPGS nanoparticles: in vitro and in vivo performance compared with Abraxane ${ }^{\circledR}$. Colloids Surf B Biointerfaces 113: 43-50, 2014.

30. Mehta G, Hsiao AY, Ingram M, Luker GD and Takayama S: Opportunities and challenges for use of tumor spheroids as models to test drug delivery and efficacy. J Control Release 164: 192-204, 2012.

31. Pampaloni F, Reynaud EG and Stelzer EH: The third dimension bridges the gap between cell culture and live tissue. Nat Rev Mol Cell Biol 8: 839-845, 2007.

32. Davies CD, Müller H, Hagen I, Gårseth $M$ and Hjelstuen MH: Comparison of extracellular matrix in human osteosarcomas and melanomas growing as xenografts, multicellular spheroids, and monolayer cultures. Anticancer Res 17: 4317-4326, 1997.

33. Kim SC, Kim DW, Shim YH, Bang JS, Oh HS, Wan Kim S and Seo MH.: In vivo evaluation of polymeric micellar paclitaxel formulation: toxicity and efficacy. J Control Release 72: 191-202, 2001.

34. Desai N, Trieu V, Yao Z, Louie L, Ci S, Yang A, Tao C, De T, Beals B, Dykes D, et al: Increased antitumor activity, intratumor paclitaxel concentrations, and endothelial cell transport of cremophor-free, albumin-bound paclitaxel, ABI-007, compared with cremophor-based paclitaxel. Clin Cancer Res 12: 1317-1324, 2006.

35. Maranhão RC, Cesar TB, Pedroso-Mariani SR, Hirata MH and Mesquita $\mathrm{CH}$ : Metabolic behavior in rats of a nonprotein microemulsion resembling low-density lipoprotein. Lipids 28 : 691-696, 1993. 
36. Bauer KR, Brown M, Cress RD, Parise CA and Caggiano V: Descriptive analysis of estrogen receptor (ER)-negative, progesterone receptor (PR)-negative, and HER2-negative invasive breast cancer, the so-called triple-negative phenotype: a population-based study from the California Cancer Registry. Cancer 109: 1721-1728, 2007.

37. Hudis CA and Gianni L: Triple-negative breast cancer: an unmet medical need. Oncologist 16 (Suppl 1): 1-11, 2011.

38. Lei H, Hofferberth SC, Liu R, Colby A, Tevis KM, Catalano P, Grinstaff MW and Colson YL: Paclitaxel-loaded expansile nanoparticles enhance chemotherapeutic drug delivery in mesothelioma 3-dimensional multicellular spheroids. J Thorac Cardiovasc Surg 149: 1417-1425, 2015.
39. Xin H, Sha X, Jiang X, Zhang W, Chen L and Fang X: Anti-glioblastoma efficacy and safety of paclitaxel-loading Angiopep-conjugated dual targeting PEG-PCL nanoparticles. Biomaterials 33: 8167-8176, 2012. 